OPEN ACCESS

Edited by:

Dan Hu,

Renmin Hospital of Wuhan University,

China

Reviewed by:

Mohit Turagam

Mount Sinai Hospital, United States

Songyun Wang,

Renmin Hospital of Wuhan University,

China

Jeffrey Shi Kai Chan,

Cardiovascular Analytics Group,

Hong Kong SAR, China

*Correspondence.

Jian Ma

majian_fuwai@126.com

Specialty section:

This article was submitted to

Cardiac Electrophysiology,

a section of the journal

Frontiers in Physiology

Received: 24 August 2021 Accepted: 27 October 2021 Published: 30 November 2021

Citation:

Li J-h, Xie H-y, Chen Y-q, Cao Z-j, Tang Q-h, Guo X-g, Sun Q and Ma J

(2021) Risk of New-Onset Atrial Fibrillation Post-cavotricuspid Isthmus

Ablation in Typical Atrial Flutter

Without History of Atrial Fibrillation.

Front. Physiol. 12:763478.

doi: 10.3389/fphys.2021.763478

\section{Risk of New-Onset Atrial Fibrillation Post-cavotricuspid Isthmus Ablation in Typical Atrial Flutter Without History of Atrial Fibrillation}

Jia-hui Li, Hai-yang Xie, Yan-qiao Chen, Zhong-jing Cao, Qing-hui Tang, Xiao-gang Guo, Qi Sun and Jian Ma*

Arrhythmia Center, State Key Laboratory of Cardiovascular Disease, Fuwai Hospital, National Center for Cardiovascular Diseases, Chinese Academy of Medical Sciences and Peking Union Medical College, Beijing, China

Aims: The aim was to describe the incidence of atrial fibrillation (AF) after cavotricuspid isthmus (CTI) ablation in patients with typical atrial flutter (AFL) without history of AF and to identify risk factors for new-onset AF after the procedure.

Methods: A total of 191 patients with typical AFL undergoing successful CTI ablation were enrolled. Patients who had history of AF, structural heart disease, cardiac surgery, or ablation or who received antiarrhythmic drug after procedure were excluded. Clinical and electrophysiological data were collected.

Results: There were 47 patients (24.6\%) developing new AF during a follow-up of $3.3 \pm 1.9$ years after CTI ablation. Receiver operating characteristic (ROC) curves indicated that the cut-off values of left atrial diameter $(L A D)$ and $\mathrm{CHA}_{2} \mathrm{DS}_{2}$-VASc score were $42 \mathrm{~mm}$ and 2, with area under the curve of 0.781 and 0.550 , respectively. The multivariable Cox regression analysis revealed that obstructive sleep apnea (OSA) [hazard ratio $(\mathrm{HR}) 3.734,95 \%$ confidence interval $(\mathrm{Cl}) 1.470-9.484, P=0.006$ ], advanced interatrial block (alAB) $(\mathrm{HR} 2.034,95 \% \mathrm{Cl} 1.017-4.067, P=0.045)$, $\mathrm{LAD}>42 \mathrm{~mm}(\mathrm{HR} 2.710,95 \% \mathrm{Cl} 1.478-4.969, P=0.001)$, and $\mathrm{CHA}_{2} \mathrm{DS}_{2}$-VASc score $>2(\mathrm{HR} 2.123,95 \% \mathrm{Cl} 1.118-4.034, P=0.021)$ were independent risk factors of new-onset $\mathrm{AF}$.

Conclusion: A combination of OSA, alAB, LAD > $42 \mathrm{~mm}$, and $\mathrm{CHA}_{2} \mathrm{DS}_{2}-\mathrm{VASc}>2$ was a strongly high risk for new-onset AF after ablation for typical AFL, and it had significance in postablation management in clinical practice.

Keywords: typical atrial flutter, atrial fibrillation, catheter ablation, advanced interatrial block (aIAB), left atrial diameter (LAD), CHA2DS2-VASc score

\section{INTRODUCTION}

Catheter ablation of cavotricuspid isthmus (CTI) is an effective procedure with high success rate for typical atrial flutter (AFL). The development of atrial fibrillation (AF) has always been a big concern after the ablation, and prediction of AF occurrence is important to optimize postablation management of AFL. Previous studies have demonstrated an incidence of AF ranging from 25 to $82 \%$ in patients with typical AFL after CTI ablation (Chinitz et al., 2007; Ellis et al., 2007; 
Voight et al., 2014; Chen et al., 2015; Celikyurt et al., 2017; Fujimoto et al., 2020). However, in most of these studies, patients with and without prior AF were both enrolled, and the history of AF was identified as the greatest risk factor for AF occurrence during the postablation period. Data on incidence and predictors of AF in patients without history of AF after typical AFL ablation are still relatively sparse.

The aim of this study was (a) to describe the incidence of AF after CTI ablation in patients with typical AFL without history of $\mathrm{AF}$ and (b) to identify risk factors for the occurrence of $\mathrm{AF}$ after CTI ablation.

\section{MATERIALS AND METHODS}

\section{Study Population}

This study involved 388 patients who were first diagnosed as typical AFL and underwent CTI ablation alone at Ward 2 of Arrhythmia Center between May 2012 and January 2020. All medical records, 12-lead electrocardiogram (ECG), and 24-h Holter obtained from enrolled patients were reviewed to exclude any atrial arrhythmias before the onset of flutter. Of the 39 patients excluded for prior history of AF, 36 had AF verified by ECG and/or 24-h Holter, and the other 3 had previous AF ablation reported by medical records. Other excluded subjects were those with previous history of cardiac surgery $(N=134)$, ablation for atrial arrhythmias $(N=11)$, structural heart disease $(N=10)$, and treatment with antiarrhythmic drugs (AADs) after ablation $(N=3)$. A total of 191 patients with typical AFL confirmed by ECG, Holter, or electrophysiological study were enrolled in final study (Figure 1). The data on baseline characteristics, echocardiographic, and electrocardiographic parameters before ablation were recorded. $\mathrm{CHA}_{2} \mathrm{DS}_{2}$-VASc score was calculated as previously (Liu et al., 2020) introduced. The study was conducted in accordance with the Declaration of Helsinki (as revised in 2013). The study was approved by the ethics committee of Fuwai Hospital, and informed consent was obtained from all the patients.

\section{Catheter Ablation}

Typical AFL was defined as regular negative sawtooth flutter waves in the inferior leads and positive flutter waves in lead V1, with a regular atrial rate between 240 and 350 beats/min. Entrainment techniques were used to determine CTI dependency. Radiofrequency ablation lesions were performed from the tricuspid valve to the inferior vena cava. The successful ablation was defined by the bidirectional CTI block after a surveillance period of at least $10 \mathrm{~min}$. Inducibility of any atrial arrhythmia after CTI ablation was attempted at the discretion of the operator, using atrial burst pacing with infusion of isoproterenol. Induced AF that lasted over $30 \mathrm{~s}$ was defined as sustained AF. If AF could not spontaneously convert to sinus rhythm, the electrical cardioversion would be performed.

\section{Electrophysiology Study}

After CTI ablation, the standard 12-lead ECGs of patients in sinus rhythm was studied by two independent observers. Advanced interatrial block (aIAB) was defined as P-wave duration $>120 \mathrm{~ms}$ with a biphasic morphology in inferior leads (Bayes de Luna et al., 2012; Figure 2). For patients without a bundle branch block, fragmented QRS (fQRS) complex was defined as an abnormal R'wave notching in the nadir of $S$ wave or the presence of more than one $\mathrm{R}^{\prime}$ in at least two consecutive leads. For patients with bundle branch block (QRS duration $>120 \mathrm{~ms}$ ), fQRS complex was defined as more than two $\mathrm{R}^{\prime}$ or $>2$ notches in $\mathrm{R}$ wave or $>2$ notches in the nadir of $\mathrm{S}$ wave in at least two consecutive leads (Das and Zipes, 2009).

\section{Periprocedural Anticoagulation Strategy}

All patients received oral anticoagulants (OACs) (e.g., warfarin, dabigatran, or rivaroxaban) for at least 3 weeks before ablation and continued for at least 1 month after the procedure. The international normalized ratio was maintained between 2 and 3 if the patient received warfarin. After 1 month, OAC was discontinued in asymptomatic patients whose ECG or Holter monitoring maintained sinus rhythm without AFL/AF recurrence. OAC was restarted in patients who had AFL/AF recurrence during the follow-up.

\section{Follow-up of Patients}

No AADs were prescribed after the procedure. All patients underwent continuous ECG monitoring for at least $24 \mathrm{~h}$ until discharge from hospital, and they were followed up at 1, 3, 6, 9, and 12 months after the procedure and every 6 months thereafter. During each follow-up or if the patient was symptomatic, 12-lead ECG or 24-h Holter recordings were obtained to monitor rhythm status. Outpatient records, diagnoses, and subsequent hospital admissions were collected using electronic medical recording system and telephone interviews. New-onset AF was defined as any episode of AF lasting $\geq 30$ s documented by 12-lead ECG or 24-h Holter monitoring (Poty et al., 1996). The clinical endpoint was new-onset AF during follow-up. The time interval was determined from ablation procedure to new occurrence of AF.

\section{Statistical Analysis}

Continuous variables were expressed as mean \pm standard deviation (SD) and compared using two-tailed $t$-test, while categorical variables were expressed as counts and percentage and compared using the Chi-square test or Fisher's exact test when appropriate. The $\mathrm{k}$ statistic was used to evaluate the interobserver agreement (IOA) for the variables measured from the ECG. A receiver operating characteristic (ROC) curve with the area under the curve (AUC) was generated, and the optimal cut-off value of the left atrial diameter (LAD) for predicting new-onset AF was obtained. Cox regression modeling was used to determine risk factors for new-onset AF after typical AFL ablation. Variables with statistical significance $(P$-value $<0.1)$ in univariable regression models were included in the multivariable regression model using "forward LR" method to determine their significance after adjustment for potential confounders. KaplanMeier curves and log-rank tests were performed to compare AF-free survival. All statistical tests were two-sided, and statistical significance was defined as $P$-value $<0.05$. All analyses were 


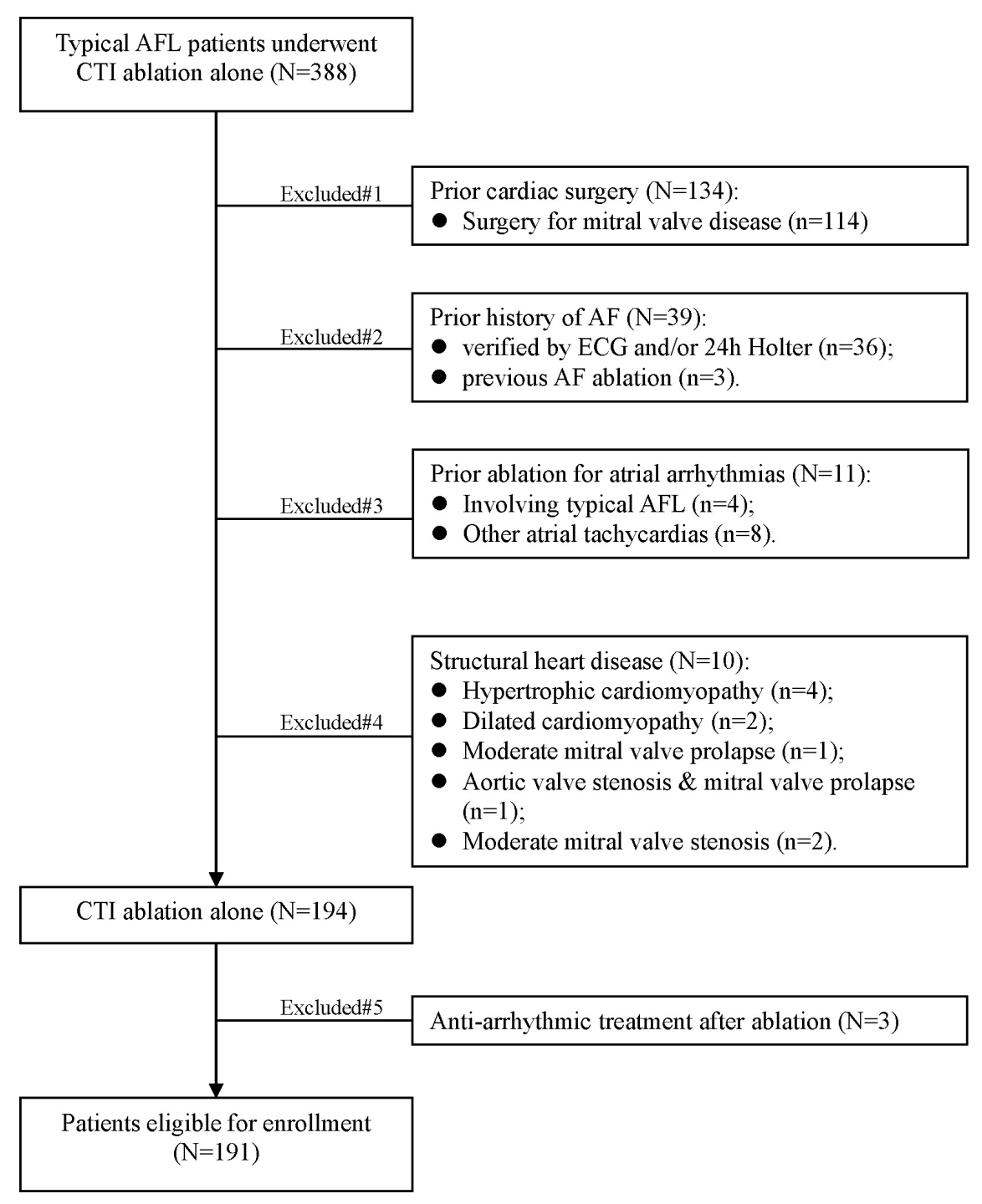

FIGURE 1 | Flowchart of this study. AF, atrial fibrillation; AFL, atrial flutter; CTI, cavotricuspid isthmus.

performed using the SPSS 24.0 software (SPSS Inc., Chicago, IL, United States).

\section{RESULTS}

A total of 191 patients were enrolled in the final analysis. Among them, the mean age was $59.0 \pm 13.8$ years, and the majority were males $(86.4 \%)$. The baseline characteristics in detail were shown in Table 1 . The bidirectional CTI block was successfully achieved without ablation complication in all 191 patients. After CTI ablation, a combination of atrial burst pacing and isoproterenol infusion was performed in eight patients, but none of them was induced with any atrial arrhythmia.

No patient was lost to follow-up in this study. Twelve patients (6.3\%) had CTI-dependent AFL recurrence at an average of
$8.6 \pm 7.3$ months after the procedure, and seven of them had recurrence within the first year $(4.1 \pm 2.7$ months $)$ after ablation. Of the 12 patients, 11 patients underwent repeat CTI ablation successfully and 3 patients eventually developed AF after repeat ablation during the follow-up.

Of the 47 patients (24.6\%) developing AF during a follow-up period of $3.3 \pm 1.9$ years, $36.2 \%(18 / 47)$ were documented within 1 year after procedure and 43.6\% (20/47) more than 2 years after procedure. Among new AF occurrences, 93.6\% (44/47) were detected by ECG or Holter monitoring and the rest $6.4 \%(3 / 47)$ by clinical follow-up only. Patients with obstructive sleep apnea (OSA) (12.8 vs. $2.1 \%, P<0.001$ ), aIAB (27.7 vs. $4.2 \%, P<0.001$ ), and larger LAD $(43.5 \pm 4.7$ vs. $38.1 \pm 5.1 \mathrm{~mm}, P<0.001)$ had higher incidence of new-onset AF (Table 1).

The ROC curves based on AF occurrence indicated that the cut-off values of LAD and $\mathrm{CHA}_{2} \mathrm{DS}_{2}$-VASc score were $42 \mathrm{~mm}$ and 2, with AUC of 0.781 and 0.550 , respectively (Figure 3). 

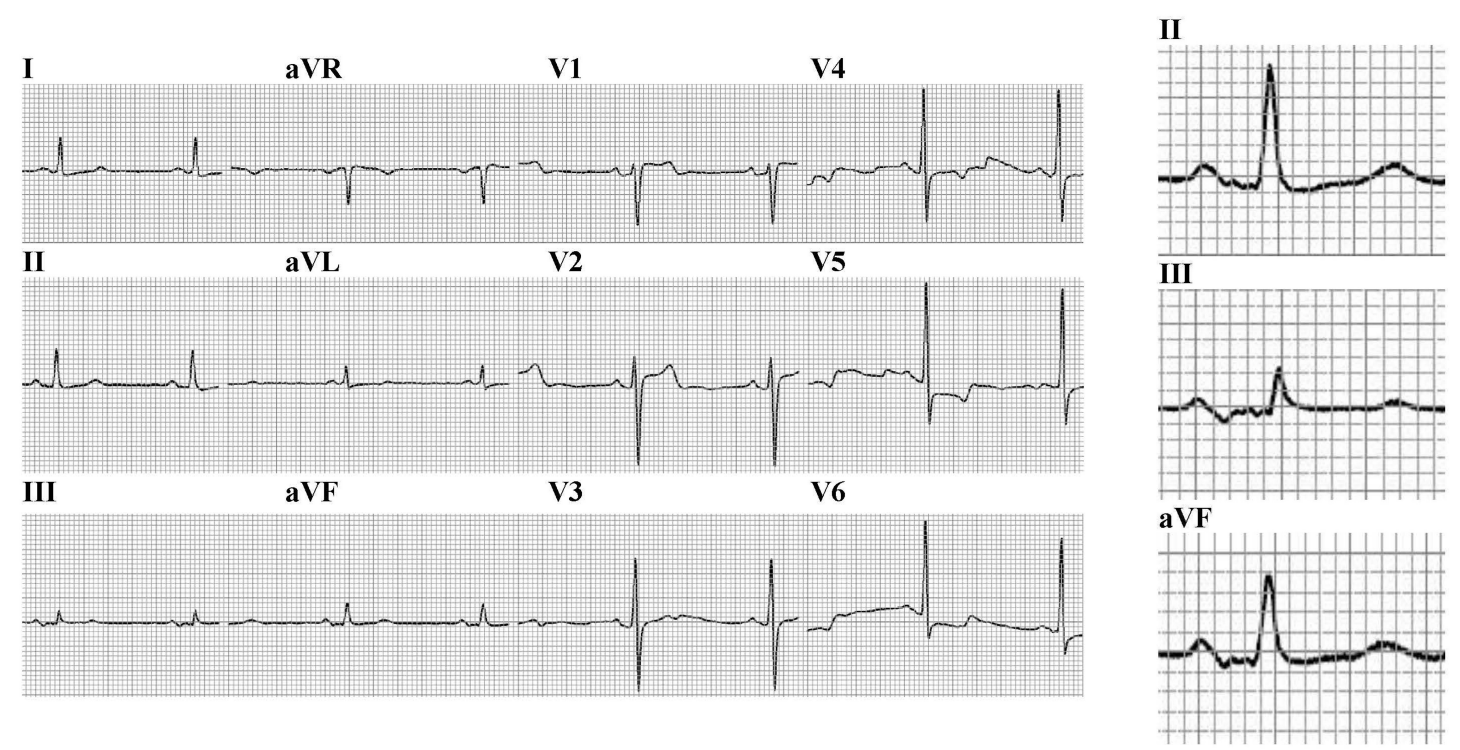

FIGURE 2 | The 12-lead electrocardiogram of a patient with advanced interatrial block (P-wave duration > $120 \mathrm{~ms}$ with a biphasic morphology in inferior leads) $(25 \mathrm{~mm} / \mathrm{s}, 10 \mathrm{~mm} / \mathrm{mV})$.

Patients with LAD > $42 \mathrm{~mm}(58 / 191,30.4 \%)$ had longer P-wave duration (132.4 \pm 19.9 vs. $125.7 \pm 18.9 \mathrm{~ms}, P=0.038)$, more aIAB (22.4 vs. $4.5 \%, P<0.001)$, and higher AF recurrence (43.1 vs. $16.5 \%, P<0.001$ ). For patients with $\mathrm{CHA}_{2} \mathrm{DS}_{2}$ VASc score $>2$, advanced age $(70.0 \pm 12.3$ vs. $56.8 \pm 13.0$, $P<0.001$ ), hypertension (57.6 vs. $36.7 \%, P=0.026$ ), diabetes (54.5 vs. $12.0 \%, P<0.001$ ), and congestive heart failure (3.0 vs. $1.9 \%, P<0.001)$ were common, and these patients were more vulnerable to AF (42.4 vs. 20.9\%, $P=0.009$ ) (Table 2 and Figure 4).

The $k$ value that evaluated IOA for aIAB between the two cardiologists was 0.90 . The presence of aIAB was observed in 19 of all 191 patients (9.9\%). Patients with aIAB had longer P-wave duration $(137.7 \pm 15.9$ vs. $126.6 \pm 19.4 \mathrm{~ms}, P=0.026)$, larger LAD (44.7 \pm 5.9 vs. $38.9 \pm 5.2 \mathrm{~mm}, P<0.001)$, and higher incidence of AF (68.4 vs. $19.8 \%, P<0.001)$ comparing to those without aIAB (Table 2).

As shown in Kaplan-Meier curves (Figure 5), patients with aIAB, LAD > $42 \mathrm{~mm}$, or higher $\mathrm{CHA}_{2} \mathrm{DS}_{2}$-VASc score had higher incidence of AF during the follow-up. Based on prior reports (Ellis et al., 2007; Voight et al., 2014; Celikyurt et al., 2017; Fujimoto et al., 2020) and clinical relevance, potential confounders were selected into the Cox regression model to determine risk factors for new-onset AF. The univariable analysis revealed that patients with OSA [hazard ratio (HR) 5.113, 95\% confidence interval (CI) 2.143-12.196, $P<0.001$ ], aIAB (HR $3.485,95 \%$ CI 1.824-6.656, $P<0.001)$, LAD $>42 \mathrm{~mm}(\mathrm{HR}$ 2.910, 95\% CI 1.637-5.171, $P<0.001)$, and $\mathrm{CHA}_{2} \mathrm{DS}_{2}$-VASc $>2$ (HR 2.287, 95\% CI 1.221-4.287, $P=0.010$ ) were at increasing risk of AF occurrence after CTI ablation. The multivariable Cox regression analysis revealed that OSA (HR 3.734, 95\% CI 1.470-9.484, $P=0.006$ ), aIAB (HR 2.034, 95\% CI 1.017-4.067, $P=0.045), \mathrm{LAD}>42 \mathrm{~mm}$ (HR 2.710, 95\% CI 1.478-4.969,
$P=0.001)$, and $\mathrm{CHA}_{2} \mathrm{DS}_{2}$-VASc $>2$ (HR 2.123, 95\% CI 1.118$4.034, P=0.021)$ were independent risk factors of new-onset $\mathrm{AF}$ after CTI ablation (Table 3 ).

\section{DISCUSSION}

The main findings of the current study included that (a) among patients with typical AFL without history of AF, nearly a quarter of them developed new-onset AF after CTI ablation and (b) OSA, aIAB, higher $\mathrm{LAD}$, and $\mathrm{CHA}_{2} \mathrm{DS}_{2}$-VASc score were independent risk factors for new-onset $\mathrm{AF}$ after the procedure.

\section{Atrial Flutter Recurrence}

In present study, the average time interval between CTI ablation and AFL recurrence was over 1 year, with the interval ranging from 3 to 74 months. The rate of AFL recurrence was $6.3 \%$ and the AFL recurrence-free rate was $>90 \%$, which was consistent with reports of previous studies (Chinitz et al., 2007; Chen et al., 2015).

\section{Atrial Fibrillation Occurrence After Cavotricuspid Isthmus Ablation}

For patients with AFL undergoing CTI ablation, the occurrence of AF has always been a concern in rhythm management after the procedure. Although the mechanism of AF development after AFL ablation has not been clearly characterized, the occurrence of these two atrial arrhythmias shares similar atrial substrate and electrophysiological triggers. Ectopic beats and focal activation from atrium or pulmonary veins lead to AF as well as the conversion from AFL to AF (Hsieh et al., 2001; Delise et al., 2006).

In present study, $24.6 \%$ of all patients developed new-onset $\mathrm{AF}$ in a mean follow-up time of 3.3 years after CTI ablation. 
This result was similar to the incidence of $25 \%$ reported by Voight et al. (2014) and 22\% reported by Celikyurt et al. (2017). However, higher incidence of AF after AFL ablation was observed in some studies. Using implanted loop recorders to monitor heart rhythm, Mittal et al. (2013) reported that nearly 50\% of enrolled patients developed AF after AFL ablation. Even higher incidence $(82 \%)$ was observed in study from Ellis et al. (2007). Part of reason for the high incidence of AF was that patients with more complicated comorbidities were enrolled in their study. Besides, a longer period of follow-up (89 months) also allowed the progression of AF, which contributes to the higher incidence.

All patients in this study underwent CTI ablation alone, and nearly $75 \%$ of them were free from atrial arrhythmia during follow-up. In view of the risk of complications, sequential iatrogenic arrhythmia and uncertain benefit of preventing AF,

TABLE 1 | Clinical characteristics of the study population.

\begin{tabular}{lcccc}
\hline Variable & Total & AF & Non-AF & $P$ \\
& $\mathbf{N = 1 9 1}$ & $\mathbf{N = 4 7}$ & $\mathbf{N = 1 4 4}$ & \\
\hline Age (year) & $59.0 \pm 13.8$ & $60.0 \pm 12.1$ & $58.7 \pm 14.3$ & 0.772 \\
Male (\%) & $165(86.4)$ & $40(85.1)$ & $125(86.8)$ & 0.768 \\
Body mass index (kg/m²) & $25.6 \pm 3.5$ & $25.3 \pm 3.2$ & $25.7 \pm 3.6$ & 0.945 \\
New-onset AF (\%) & $47(24.6)$ & - & - & - \\
Comorbidities & & & & \\
Hypertension (\%) & $77(40.3)$ & $14(29.8)$ & $63(43.8)$ & 0.090 \\
Diabetes mellitus (\%) & $37(19.4)$ & $8(17.0)$ & $29(20.1)$ & 0.639 \\
Obstructive sleep apnea (\%) & $9(4.7)$ & $6(12.8)$ & $3(2.1)$ & 0.009 \\
Ischemic heart disease (\%) & $15(7.9)$ & $3(6.4)$ & $12(8.3)$ & 0.905 \\
Congestive heart failure (\%) & $4(2.1)$ & $1(2.1)$ & $3(2.1)$ & 1.000 \\
Thyroid disease* & $4(2.1)$ & $2(4.3)$ & $2(1.4)$ & 0.545 \\
Previous stroke/TIA & $4(2.1)$ & $2(4.3)$ & $2(1.4)$ & 0.545
\end{tabular}

\section{Electrocardiographic parameters}

P-wave duration (ms) $\quad 127.7 \pm 19.4 \quad 128.3 \pm 18.3127 .5 \pm 19.80 .982$

Right bundle branch block (\%) $\quad 30(15.7) \quad 8(17.0) \quad 22(15.3) \quad 0.775$

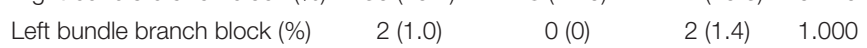

QTc interval (ms) $\quad 432.6 \pm 40.2 \quad 433.6 \pm 44.8432 .2 \pm 38.70 .779$

Fragmented QRS complex (\%) $32(16.8) \quad 12(25.5) \quad 20(13.9) \quad 0.063$

$\begin{array}{lllll}\text { Advanced interatrial block (\%) } \quad 19(9.9) & 13(27.7) & 6(4.2) & <0.001\end{array}$

Echocardiographic parameters

$\begin{array}{lcccc}\text { LVEF (\%) } & 58.7 \pm 8.2 & 58.1 \pm 8.1 & 58.9 \pm 8.2 & 0.448 \\ \text { Left atrial diameter (mm) } & 39.4 \pm 5.5 & 43.5 \pm 4.7 & 38.1 \pm 5.1 & <0.001 \\ \text { LAD > 42mm (\%) } & 58(30.4) & 25(53.2) & 33(22.9) & <0.001 \\ \text { Enlarged right atrium (\%) } & 35(18.3) & 12(25.5) & 23(16.0) & 0.141 \\ \text { CHA }_{2} \text { DS }_{2} \text {-Vasc score } & 1.4 \pm 1.4 & 1.7 \pm 1.7 & 1.3 \pm 1.2 & 0.262 \\ \text { Concomitant drug use } & & & & \\ \text { ACEi/ARB (\%) } & 61(31.9) & 10(21.3) & 51(35.4) & 0.071 \\ \beta \text {-Blockers (\%) } & 112(58.6) & 26(55.3) & 86(59.7) & 0.595 \\ \text { Follow-up (months) } & 40.2 \pm 23.3 & 25.3 \pm 24.2 & 45.0 \pm 21.0<0.001\end{array}$

Data are presented as mean $\pm S D$ or $n(\%)$.

$A C E i$, angiotensin-converting enzyme inhibitor; $A F$, atrial fibrillation; $A R B$, angiotensin receptor blocker; $L A D$, left atrial diameter; $L V E F$, left ventricular ejection fraction; TIA, transient ischemic attack.

${ }^{*} A$ ll the four patients with history of hypothyroidism had normal level of thyroid function at present.

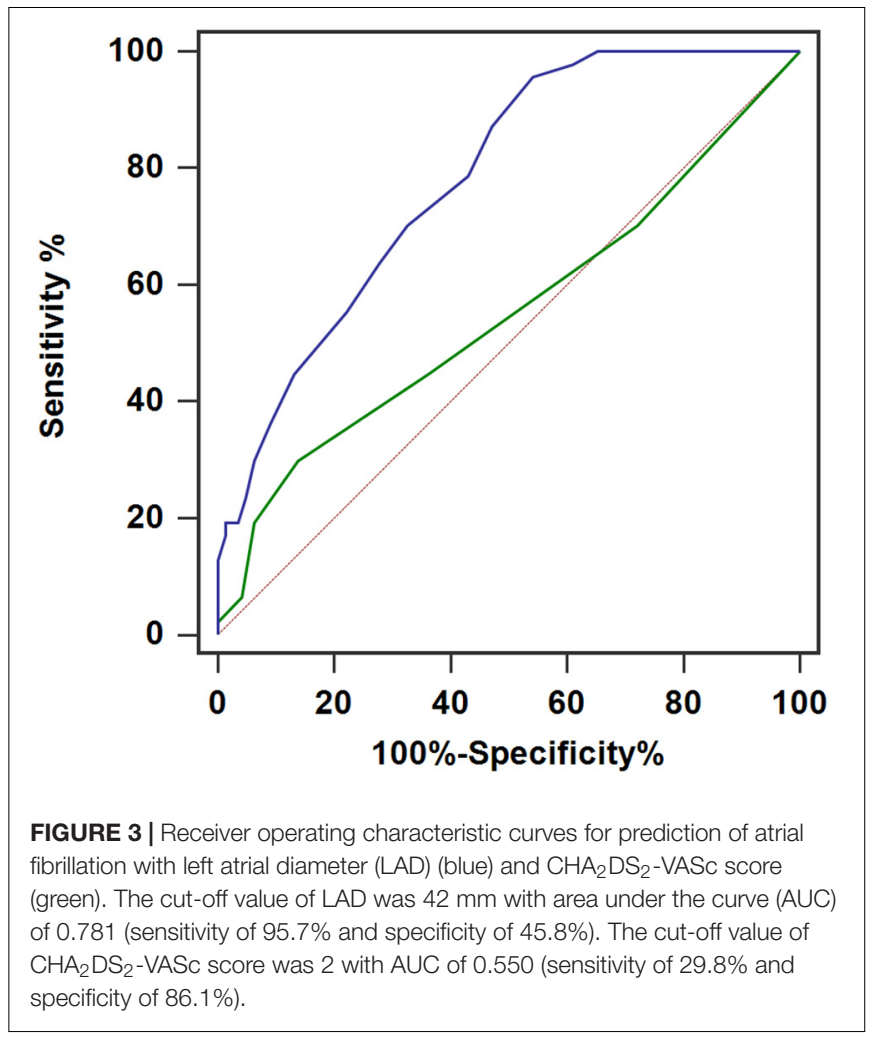

prophylactic AF ablation including pulmonary vein isolation (PVI) was not routinely performed in this study, although the pulmonary vein was confirmed to have an essential role in occurrence of AF in patients with AFL (Steinberg et al., 2014; Schneider et al., 2015). Some reports (Steinberg et al., 2014; Mohanty et al., 2015; Koerber et al., 2019) demonstrated that for typical AFL patients without history of AF, CTI ablation combined with prophylactic PVI resulted in a substantial reduction in new-onset AF after procedure. However, the risk/benefit ratio of prophylactic PVI still remained controversial. Gula et al. (2016) reported greater procedural risk and higher cost for patients who underwent ablation of CTI + PVI for isolated AFL. In current study, it was confirmed that patients with OSA, aIAB, higher LAD ( $>42 \mathrm{~mm})$, and $\mathrm{CHA}_{2} \mathrm{DS}_{2}$-VASc score $(>2)$ were at significantly increasing risk of developing new-onset AF after CTI ablation for typical AFL. For these patients, active prophylactic measures brought more benefits in preventing AF occurrence, and it might be reasonable to perform PVI combined with CTI ablation. More studies evaluating the risk/benefit ratio of prophylactic PVI combined with CTI ablation were needed to confirm this assumption.

\section{Risk Factors for New-Onset Atrial Fibrillation}

Previous evidence (Gottlieb et al., 2010; Redline et al., 2010) had shown that sleep disordered breathing increased the incidence of AF. As expected, OSA was identified as an independent risk factor for new-onset AF after ablation for typical AFL. Multiple 
mechanisms including atrial stretch, autonomic dysregulation, and abnormalities of gas exchange were raised to explain the relationship between AF and OSA (Nalliah et al., 2016). Furthermore, Nalliah et al. (2021) evaluated the left atrial substrate of AF patients with history of OSA by means of highdensity mapping, and they found that high severity of OSA correlated with increasingly remodeled left atria, which presented with lower bipolar voltage and greater voltage/conduction heterogeneity. The electroanatomical alternations significantly promoted the development of AF. These findings suggested the importance of effective treatment of sleep disordered breathing in patients with AFL after ablation.

Although the presence of IAB is not rare in clinical work (O'Neal et al., 2016), it has been widely neglected and under-recognized (Baranchuk and Bayés de Luna, 2015). In patients with constructive abnormalities including surgical scar, fibrosis, and infarction, the conduction across the Bachmann region is delayed, while the atrial septum and/or coronary sinus plays main roll in interatrial conduction (Lemery et al., 2004; Tapanainen et al., 2009). Therefore, the left atrium is activated through the inferior interatrial pathway, and a prolonged and biphasic $\mathrm{P}$ wave presents in inferior leads. In this study, the presence of $\mathrm{aIAB}$, which was defined as biphasic $\mathrm{P}$ waves with duration $>120 \mathrm{~ms}$ in inferior leads, was identified as an independent risk factor for new-onset $\mathrm{AF}$ in patients after AFL ablation. IAB was reported linking with a delayed and asynchronous activation of left atrium (Agarwal et al., 2003; Budeus et al., 2005), and it was significantly associated with AF occurrence with a HR of 2.01 (Tse et al., 2018). Also, it was a risk factor for AF recurrence after ablation (Enriquez et al., 2014;

TABLE 2 | Comparison between patients grouped by alAB, LAD, and $\mathrm{CHA}_{2} \mathrm{DS}_{2}$-Vasc score.

\begin{tabular}{|c|c|c|c|c|c|c|c|c|c|}
\hline Variable & $\begin{array}{l}\text { alAB }+ \\
N=19\end{array}$ & $\begin{array}{l}\text { alAB- } \\
N=172\end{array}$ & $P^{*}$ & $\begin{array}{c}\text { LAD }>42 \mathrm{~mm} \\
\qquad N=58\end{array}$ & $\begin{aligned} \mathrm{LAD} & \leq 42 \mathrm{~mm} \\
N & =133\end{aligned}$ & $\boldsymbol{P}^{\S}$ & $\begin{array}{c}\mathrm{CHA}_{2} \mathrm{DS}_{2}-\text { Vasc }>2 \\
N=33\end{array}$ & $\begin{array}{c}\mathrm{CHA}_{2} \mathrm{DS}_{2}-\text { Vasc } \leq 2 \\
N=158\end{array}$ & $P^{\#}$ \\
\hline Age (year) & $64.4 \pm 13.5$ & $58.4 \pm 13.8$ & 0.055 & $59.0 \pm 13.7$ & $59.0 \pm 13.9$ & 0.894 & $70.0 \pm 12.3$ & $56.8 \pm 13.0$ & $<0.001$ \\
\hline Male (\%) & $14(73.7)$ & $151(87.8)$ & 0.177 & $51(87.9)$ & $114(85.7)$ & 0.681 & $29(87.9)$ & $136(86.1)$ & 0.784 \\
\hline BMI $\left(\mathrm{kg} / \mathrm{m}^{2}\right)$ & $26.4 \pm 4.0$ & $25.5 \pm 3.4$ & 0.207 & $26.5 \pm 3.6$ & $25.2 \pm 3.4$ & 0.059 & $26.0 \pm 3.0$ & $25.5 \pm 3.6$ & 0.238 \\
\hline New-onset AF (\%) & $13(68.4)$ & $34(19.8)$ & $<0.001$ & $25(43.1)$ & $22(16.5)$ & $<0.001$ & $14(42.4)$ & $33(20.9)$ & 0.009 \\
\hline \multicolumn{10}{|l|}{ Comorbidities } \\
\hline Hypertension (\%) & $9(47.4)$ & $68(39.5)$ & 0.509 & $27(46.6)$ & $50(37.6)$ & 0.246 & $19(57.6)$ & $58(36.7)$ & 0.026 \\
\hline Diabetes (\%) & $5(26.3)$ & 32 (18.6) & 0.616 & $13(22.4)$ & $24(18.0)$ & 0.482 & $18(54.5)$ & $19(12.0)$ & $<0.001$ \\
\hline OSA (\%) & $3(15.8)$ & $6(3.5)$ & 0.067 & $3(5.2)$ & $6(4.5)$ & 1.000 & $3(9.1)$ & $6(3.8)$ & 0.393 \\
\hline $\operatorname{IHD}(\%)$ & $3(15.8)$ & $12(7.0)$ & 0.365 & $6(10.3)$ & $9(6.8)$ & 0.580 & $5(15.2)$ & $10(6.3)$ & 0.175 \\
\hline $\mathrm{CHF}(\%)$ & $0(0)$ & $4(2.3)$ & 1.000 & $3(5.2)$ & $1(0.8)$ & 0.158 & $1(3.0)$ & $3(1.9)$ & $<0.001$ \\
\hline Previous stroke/TIA (\%) & $0(0)$ & $4(2.3)$ & 1.000 & $1(1.7)$ & $3(2.3)$ & 1.000 & $2(6.1)$ & $2(1.3)$ & 0.280 \\
\hline Thyroid disease $^{\dagger}(\%)$ & $2(10.5)$ & $2(1.2)$ & 0.063 & $1(1.7)$ & $3(2.3)$ & 1.000 & $2(6.1)$ & $2(1.3)$ & 0.280 \\
\hline \multicolumn{10}{|c|}{ Electrocardiographic parameters } \\
\hline P-wave duration (ms) & $137.7 \pm 15.9$ & $126.6 \pm 19.4$ & 0.026 & $132.4 \pm 19.9$ & $125.7 \pm 18.9$ & 0.038 & $129.8 \pm 21.4$ & $127.4 \pm 19.0$ & 0.367 \\
\hline RBBB (\%) & $2(10.5)$ & $28(16.3)$ & 0.748 & $7(12.1)$ & $23(17.3)$ & 0.362 & $6(18.2)$ & $24(15.2)$ & 0.667 \\
\hline LBBB (\%) & $0(0)$ & $2(1.2)$ & 1.000 & $1(1.7)$ & $1(0.8)$ & 1.000 & $1(3.0)$ & $1(0.6)$ & 0.772 \\
\hline QTc interval (ms) & $440.4 \pm 41.0$ & $431.7 \pm 40.1$ & 0.468 & $439.3 \pm 46.8$ & $429.6 \pm 36.7$ & 0.168 & $438.7 \pm 41.1$ & $431.3 \pm 40.0$ & 0.406 \\
\hline fQRS complex (\%) & 5 (26.3) & $27(15.7)$ & 0.394 & $12(20.7)$ & $20(15.0)$ & 0.336 & $8(24.2)$ & $24(15.2)$ & 0.205 \\
\hline alAB (\%) & - & - & - & $13(22.4)$ & $6(4.5)$ & $<0.001$ & $3(9.1)$ & $16(10.1)$ & 0.857 \\
\hline \multicolumn{10}{|c|}{ Echocardiographic parameters } \\
\hline LVEF (\%) & $59.4 \pm 6.9$ & $58.6 \pm 8.3$ & 0.662 & $56.4 \pm 11.2$ & $59.7 \pm 6.3$ & 0.174 & $59.2 \pm 8.0$ & $58.6 \pm 8.2$ & 0.544 \\
\hline LAD (mm) & $44.7 \pm 5.9$ & $38.9 \pm 5.2$ & $<0.001$ & $45.8 \pm 3.1$ & $36.7 \pm 3.8$ & $<0.001$ & $41.0 \pm 5.0$ & $39.1 \pm 5.6$ & 0.088 \\
\hline LAD > 42 mm (\%) & $13(68.4)$ & $45(26.2)$ & $<0.001$ & - & - & - & $11(33.3)$ & $47(29.7)$ & 0.684 \\
\hline Enlarged RA (\%) & $4(21.1)$ & $31(18.0)$ & 0.991 & $15(25.9)$ & $20(15.0)$ & 0.075 & $10(30.3)$ & $25(15.8)$ & 0.051 \\
\hline $\mathrm{CHA}_{2} \mathrm{DS}_{2}$-Vasc score & $1.8 \pm 1.7$ & $1.4 \pm 1.3$ & 0.253 & $1.5 \pm 1.3$ & $1.4 \pm 1.4$ & 0.323 & $3.8 \pm 1.0$ & $1.0 \pm 0.8$ & $<0.001$ \\
\hline \multicolumn{10}{|l|}{ Concomitant drug use } \\
\hline ACEi/ARB (\%) & $7(36.8)$ & $54(31.4)$ & 0.629 & $24(41.4)$ & $37(27.8)$ & 0.065 & $15(45.5)$ & $46(29.1)$ & 0.067 \\
\hline$\beta$-Blockers (\%) & $11(57.9)$ & $101(58.7)$ & 0.945 & $40(69.0)$ & $72(54.1)$ & 0.056 & $21(63.6)$ & $91(57.6)$ & 0.522 \\
\hline Follow-up (months) & $42.3 \pm 30.0$ & $39.9 \pm 22.6$ & 0.673 & $36.6 \pm 23.8$ & $41.7 \pm 23.0$ & 0.142 & $36.8 \pm 24.1$ & $40.9 \pm 23.2$ & 0.402 \\
\hline
\end{tabular}

Data are presented as mean $\pm S D$ or $n(\%)$.

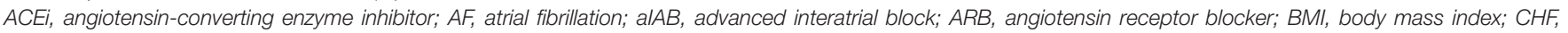

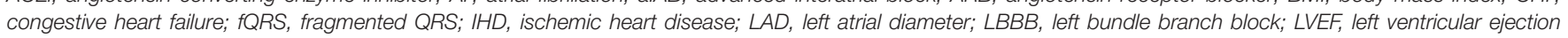
fraction; OSA, obstructive sleep apnea; RA, right atrium; RBBB, right bundle branch block; TIA, transient ischemic attack.

${ }^{*} P$ refers to comparison between patients with and without advanced interatrial block.

$₫ P$ refers to comparison between patients with $L A D>42 \mathrm{~mm}$ and with $L A D \leq 42 \mathrm{~mm}$.

${ }^{\#} P$ refers to comparison between patients with $\mathrm{CHA}_{2} D S_{2}$-VasC $>2$ and with $\mathrm{CHA}_{2} D S_{2}-$ Vasc $\leq 2$.

${ }^{\dagger} A l l$ the four patients with history of hypothyroidism had normal level of thyroid function at present. 


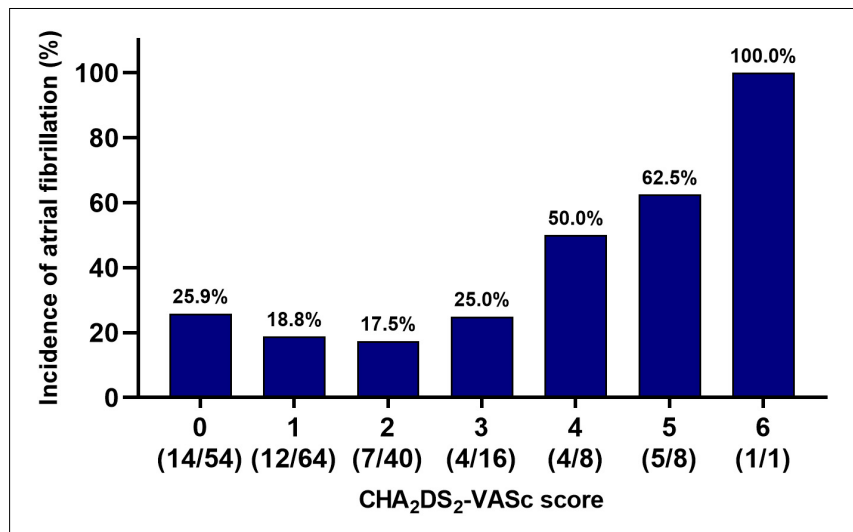

FIGURE 4 | Incidence of new-onset atrial fibrillation and $\mathrm{CHA}_{2} \mathrm{DS}_{2}$-VASc score. The incidence of new-onset $A F$ increased with an increase of $\mathrm{CHA}_{2} \mathrm{DS}_{2}$-VASc score, especially in patients with $\mathrm{CHA}_{2} \mathrm{DS}_{2}-\mathrm{VASc}>2$ $(P=0.046)$.

Fujimoto et al., 2018). Based on previous assumption, there might be a developing progress of atrial cardiomyopathy, starting from partial IAB in early stage to $\mathrm{IIAB}$ in middle stage, and eventually to developing AF. The mechanism for aIAB causing AF might involve electroanatomical remodeling, which resulted from continuous interatrial desynchrony, electrical inhomogeneity, and abnormal activation of left atrium (Fujimoto et al., 2018).

In recent years, the ability of $\mathrm{CHA}_{2} \mathrm{DS}_{2}$-VASc score had been beyond evaluating thromboembolic risk in patients with non-valvular AF, and it was helpful in various conditions, for example, reflecting atrial electroanatomical remodeling, which was associated with development of AF (Park et al., 2011). In present study, patients with $\mathrm{CHA}_{2} \mathrm{DS}_{2}$-VASc score $>2$ had advance age, greater incidence of hypertension, and diabetes mellitus and larger LAD. This result implied that patients with higher $\mathrm{CHA}_{2} \mathrm{DS}_{2}$-VASc score were more likely to undergo electroanatomical alterations, which improved development of AF. To discriminate patients between high and low risk for AF following AFL ablation, $\mathrm{CHA}_{2} \mathrm{DS}_{2}$-VASc score would be a feasible choice. Besides, $\mathrm{CHA}_{2} \mathrm{DS}_{2}$-VASc score was useful for stratifying stroke risk following AFL ablation, and $\geq 2$ was associated with higher risk of stroke after ablation (Jin et al., 2018). These findings combined with ours suggested a strong ability of $\mathrm{CHA}_{2} \mathrm{DS}_{2}$-VASc score in risk stratification and monitoring of AF, as well as prevention of thromboembolism after AFL ablation.

\section{Other Parameters}

The fQRS complex, which reflected inhomogeneous conduction and delayed activation in myocardium (Das et al., 2007), was previously reported as a risk factor for new-onset AF after AFL ablation (Fujimoto et al., 2020). In this study, it had no significance in predicting AF after CTI ablation. The mechanism of $\mathrm{fQRS}$ on AF development needs further research.

In terms of the drug use, higher angiotensin-converting enzyme inhibitor (ACEi)/angiotensin receptor blocker (ARB) usage was seen in non-AF group although no significant

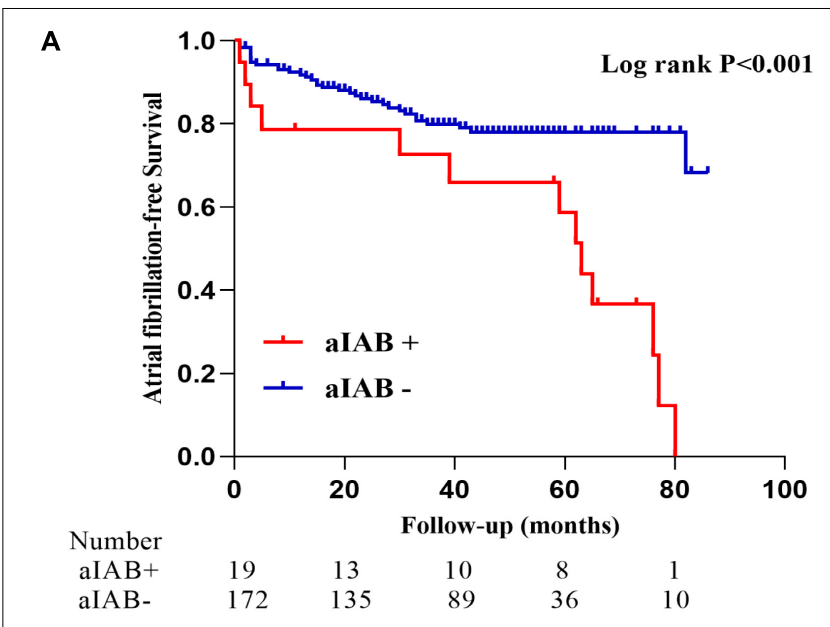

B

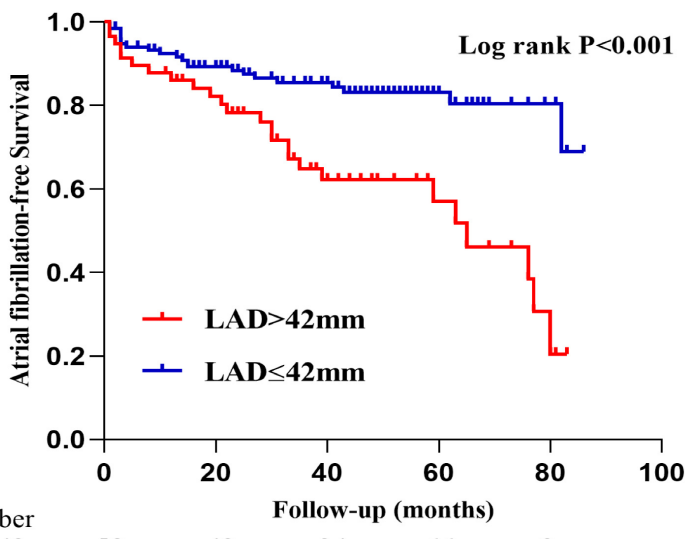

Number

$\begin{array}{lccccc}\mathrm{LAD}>42 \mathrm{~mm} & 58 & 42 & 24 & 11 & 3 \\ \mathrm{LAD} \leq 42 \mathrm{~mm} & 133 & 106 & 75 & 33 & 8\end{array}$

C

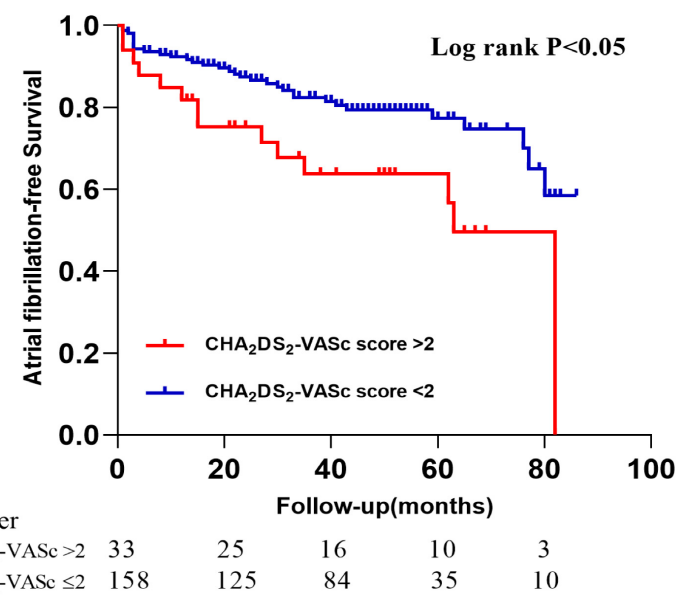

FIGURE 5 | Kaplan-Meier curves of atrial fibrillation-free survival in patients grouped by advanced interatrial block (A), left atrial diameter (B), and $\mathrm{CHA}_{2} \mathrm{DS}_{2}$-VASc score (C).

difference was found. It suggested a potential effect of ACEi/ARBs in preventing the development of postablation $\mathrm{AF}$, as previous studies (Anne et al., 2004) reported. 
TABLE 3 | Univariable and multivariable Cox regression analysis for atrial fibrillation occurrence.

\begin{tabular}{|c|c|c|c|c|c|c|}
\hline & & Univariable analysis & & & Multivariable analysis & \\
\hline Variable & HR & $95 \% \mathrm{Cl}$ & $\boldsymbol{P}$ & HR & $95 \% \mathrm{Cl}$ & $\boldsymbol{P}$ \\
\hline P-wave duration & 1.002 & $0.989-1.016$ & 0.732 & & & \\
\hline $\mathrm{al} A \mathrm{~B}$ & 3.485 & $1.824-6.656$ & $<0.001$ & 2.034 & $1.017-4.067$ & 0.045 \\
\hline $\mathrm{LAD}>42 \mathrm{~mm}$ & 2.910 & $1.637-5.171$ & $<0.001$ & 2.710 & $1.478-4.969$ & 0.001 \\
\hline Fragmented QRS & 1.652 & $0.854-3.192$ & 0.136 & & & \\
\hline QTc interval & 1.002 & $0.995-1.009$ & 0.585 & & & \\
\hline Hypertension & 0.735 & $0.391-1.380$ & 0.338 & & & \\
\hline Obstructive sleep apnea & 5.113 & $2.143-12.196$ & $<0.001$ & 3.734 & $1.470-9.484$ & 0.006 \\
\hline Body mass index & 0.391 & $0.871-1.055$ & 0.391 & & & \\
\hline RA enlarged & 1.114 & $0.570-2.174$ & 0.753 & & & \\
\hline $\mathrm{CHA}_{2} \mathrm{DS}_{2}-\mathrm{Vasc}>2$ & 2.287 & $1.221-4.287$ & 0.010 & 2.123 & $1.118-4.034$ & 0.021 \\
\hline
\end{tabular}

alAB, advanced interatrial block; $C l$, confidence interval; HR, hazard ratio; $L A D$, left atrial diameter; $R A$, right atrium.

\section{Clinical Implication}

Patients are still continuously at risk of AF occurrence and thromboembolic events in spite of undergoing successful ablation for typical AFL. Postablation management of high-risk patients has always been a big concern in clinical practice. In present study, the clinical value of $\mathrm{CHA}_{2} \mathrm{DS}_{2}$-VASc score was extended to evaluation of the risk stratification of new-onset AF. Patients with highest risk, who had OSA, aIAB, LAD > 42mm, and $\mathrm{CHA}_{2} \mathrm{DS}_{2}$-VASc score $>2$, were strongly predisposed to AF occurrence after CTI ablation. These patients should attach importance to the likelihood of AF occurrence, and a rhythm recording device (e.g., implanted loop recorder) was necessary to detect AF occurrence as soon as possible. However, the implanted loop recorder might not be easily accessible in many medical centers. In this condition, more vigilant surveillance, such as symptom evaluation, heart structural assessment, and regular rhythm monitoring, were urgently needed for patients at high risk. Additionally, it might be reasonable to perform PVI combined with CTI ablation for high-risk patients during the AFL procedure, in order to acquire more benefits in preventing AF occurrence.

The risk stratification, which was assessed by a combination of risk factors in present study, also had guiding significance in anticoagulation strategy for patients after AFL ablation. The prevalent practice in clinical work is to withdraw OCA 1 month after AFL ablation, if the patient maintains sinus rhythm without recurrence of atrial arrhythmias (Chinitz et al., 2007; Tomson et al., 2012). Therefore, many patients, especially those with multiple comorbidities, are exposed to risk of thromboembolic complications due to a lack of risk-evaluating tools and effective anticoagulation. Previous study (Jin et al., 2018) demonstrated an increasing trend in stroke with ascending $\mathrm{CHA}_{2} \mathrm{DS}_{2}$-VASc score in patients following AFL ablation. Along with findings of current study, it demonstrated that for patients undergoing CTI ablation for typical AFL, it was better to identify patients at high risk in new-onset $\mathrm{AF}$ as the first step and then to formulate an anticoagulation strategy with a closer follow-up after the procedure.

\section{Limitations}

Several limitations in this study should be addressed. First, the generalizability of results was limited because of the small number of subjects from only one single center. Besides, male accounted majority of the enrolled subjects (86.4\%), limiting the generalizability to female. Second, although ECGs and Holter records were carefully evaluated before ablation and regularly performed during follow-up period, heart rhythm monitoring was not documented completely due to a lack of reliable recording devices such as implanted loop recorders, thus the silent AFL or AF might be neglected. Third, previous study (Joza et al., 2014) reported that the inducibility of AF after typical AFL ablation was identified as a risk factor for future AF in patients with no history of AF. However, in this study, the inducibility after CTI ablation was not routinely performed, and atrial burst pacing with infusion of isoproterenol was only attempted in few patients, which limited the evaluation of atrial burst inducibility in $\mathrm{AF}$ occurrence after AFL ablation. Finally, the continuation of anticoagulation after AFL ablation depended on rhythm status and AFL/AF recurrence, and it was not guided by an evaluation tool containing risk factors in present study. Also, the incidence of AF-related thromboembolism was not documented in present study. Therefore, the anticoagulation outcome in different risk stratifications of new-onset AF following AFL ablation was needed in further study.

\section{DATA AVAILABILITY STATEMENT}

The original contributions presented in the study are included in the article/supplementary material, further inquiries can be directed to the corresponding author.

\section{ETHICS STATEMENT}

The studies involving human participants were reviewed and approved by the Ethics Committee of Fuwai Hospital. 
The patients/participants provided their written informed consent to participate in this study.

\section{AUTHOR CONTRIBUTIONS}

J-HL, H-YX, and Q-HT collected the patient data. J-HL analyzed the data and was a major contributor in writing this manuscript.

\section{REFERENCES}

Agarwal, Y. K., Aronow, W. S., Levy, J. A., and Spodick, D. H. (2003). Association of interatrial block with development of atrial fibrillation. Am. J. Cardiol. 91:882.

Anne, W., Willems, R., Van der Merwe, N., Van de Werf, F., Ector, H., and Heidbuchel, H. (2004). Atrial fibrillation after radiofrequency ablation of atrial flutter: preventive effect of angiotensin converting enzyme inhibitors, angiotensin II receptor blockers, and diuretics. Heart 90, 1025-1030. doi: 10.1136/hrt.2003.023069

Baranchuk, A., and Bayés de Luna, A. (2015). The P-wave morphology: what does it tell us? Herzschrittmacherther. Elektrophysiol. 26, 192-199.

Bayes de Luna, A., Platonov, P., Cosio, F. G., Cygankiewicz, I., Pastore, C., Baranowski, R., et al. (2012). Interatrial blocks. A separate entity from left atrial enlargement: a consensus report. J. Electrocardiol. 45, 445-451. doi: 10.1016/j. jelectrocard.2012.06.029

Budeus, M., Hennersdorf, M., Perings, C., Wieneke, H., Erbel, R., and Sack, S. (2005). Prediction of the recurrence of atrial fibrillation after successful cardioversion with P wave signal-averaged ECG. Ann. Noninvasive Electrocardiol. 10, 414-419. doi: 10.1111/j.1542-474X.2005.00059.x

Celikyurt, U., Knecht, S., Kuehne, M., Reichlin, T., Muehl, A., Spies, F., et al. (2017). Incidence of new-onset atrial fibrillation after cavotricuspid isthmus ablation for atrial flutter. Europace 19, 1776-1780. doi: 10.1093/europace/euw343

Chen, K., Bai, R., Deng, W., Gao, C., Zhang, J., Wang, X., et al. (2015). HATCH score in the prediction of new-onset atrial fibrillation after catheter ablation of typical atrial flutter. Heart Rhythm 12, 1483-1489. doi: 10.1016/j.hrthm.2015. 04.008

Chinitz, J. S., Gerstenfeld, E. P., Marchlinski, F. E., and Callans, D. J. (2007). Atrial fibrillation is common after ablation of isolated atrial flutter during long-term follow-up. Heart Rhythm 4, 1029-1033. doi: 10.1016/j.hrthm.2007.04.002

Das, M. K., Saha, C., El Masry, H., Peng, J., Dandamudi, G., Mahenthiran, J., et al. (2007). Fragmented QRS on a 12-lead ECG: a predictor of mortality and cardiac events in patients with coronary artery disease. Heart Rhythm 4, 1385-1392. doi: 10.1016/j.hrthm.2007.06.024

Das, M. K., and Zipes, D. P. (2009). Fragmented QRS: a predictor of mortality and sudden cardiac death. Heart Rhythm 6, S8-S14.

Delise, P., Sitta, N., Coro, L., Marras, E., Sciarra, L., Bocchino, M., et al. (2006). Common atrial flutter and atrial fibrillation are not always two stages of the same disease. A long-term follow-up study in patients with atrial flutter treated with cavo-tricuspid isthmus ablation. J. Cardiovasc. Med. (Hagerstown) 7, 800-805. doi: 10.2459/01.jcm.0000250867.33036.fc

Ellis, K., Wazni, O., Marrouche, N., Martin, D., Gillinov, M., McCarthy, P., et al. (2007). Incidence of atrial fibrillation post-cavotricuspid isthmus ablation in patients with typical atrial flutter: left-atrial size as an independent predictor of atrial fibrillation recurrence. J. Cardiovasc. Electrophysiol. 18, 799-802. doi: 10.1111/j.1540-8167.2007.00885.x

Enriquez, A., Conde, D., Hopman, W., Mondragon, I., Chiale, P. A., de Luna, A. B., et al. (2014). Advanced interatrial block is associated with recurrence of atrial fibrillation post pharmacological cardioversion. Cardiovasc. Ther. 32, 52-56. doi: 10.1111/1755-5922.12063

Fujimoto, Y., Yodogawa, K., Maru, Y. J., Oka, E., Hayashi, H., Yamamoto, T., et al. (2018). Advanced interatrial block is an electrocardiographic marker for recurrence of atrial fibrillation after electrical cardioversion. Int. J. Cardiol. 272, 113-117. doi: 10.1016/j.ijcard.2018.07.135

Fujimoto, Y., Yodogawa, K., Oka, E., Hayashi, H., Yamamoto, T., Murata, H., et al. (2020). Significance of fragmented QRS complexes for predicting new-onset atrial fibrillation after cavotricuspid isthmus-dependent atrial flutter ablation. Heart Rhythm 17, 1493-1499. doi: 10.1016/j.hrthm.2020.04.021
Y-QC, Z-JC, X-GG, and QS took the revision of this manuscript. All authors agreed to be accountable for the content of the work.

\section{FUNDING}

This study was supported by the National Natural Science Foundation of China (Grant No. 81670309).

Gottlieb, D. J., Yenokyan, G., Newman, A. B., O’Connor, G. T., Punjabi, N. M., Quan, S. F., et al. (2010). Prospective study of obstructive sleep apnea and incident coronary heart disease and heart failure: the sleep heart health study. Circulation 122, 352-360. doi: 10.1161/circulationaha.109.901801

Gula, L. J., Skanes, A. C., Klein, G. J., Jenkyn, K. B., Redfearn, D. P., Manlucu, J., et al. (2016). Atrial flutter and atrial fibrillation ablation - sequential or combined? A cost-benefit and risk analysis of primary prevention pulmonary vein ablation. Heart Rhythm 13, 1441-1448. doi: 10.1016/j.hrthm.2016.02.018

Hsieh, M. H., Tai, C. T., Tsai, C. F., Yu, W. C., Lin, W. S., Huang, J. L., et al. (2001). Mechanism of spontaneous transition from typical atrial flutter to atrial fibrillation: role of ectopic atrial fibrillation foci. Pacing Clin. Electrophysiol. 24, 46-52. doi: 10.1046/j.1460-9592.2001.00046.x

Jin, M. N., Song, C., Kim, T. H., Uhm, J. S., Pak, H. N., Lee, M. H., et al. (2018). CHA(2)DS(2)-VASc Score in the Prediction of Ischemic Stroke in Patients after Radiofrequency Catheter Ablation of Typical Atrial Flutter. Yonsei Med. J. 59, 236-242. doi: 10.3349/ymj.2018.59.2.236

Joza, J., Filion, K. B., Eberg, M., Proietti, R., Nascimento, T., Bernier, M., et al. (2014). Prognostic value of atrial fibrillation inducibility after right atrial flutter ablation. Heart Rhythm 11, 1870-1876. doi: 10.1016/j.hrthm.2014.06.032

Koerber, S. M., Turagam, M. K., Gautam, S., Winterfield, J., Wharton, J. M., Lakkireddy, D., et al. (2019). Prophylactic pulmonary vein isolation during cavotricuspid isthmus ablation for atrial flutter: a meta-analysis. Pacing Clin. Electrophysiol. 42, 493-498. doi: 10.1111/pace.13637

Lemery, R., Soucie, L., Martin, B., Tang, A. S., Green, M., and Healey, J. (2004). Human study of biatrial electrical coupling: determinants of endocardial septal activation and conduction over interatrial connections. Circulation 110, 20832089. doi: 10.1161/01.CIR.0000144461.83835.A1

Liu, F., Xin, Z., Bin Waleed, K., Lin, Y., Tse, G., Luhanga, A., et al. (2020). $\mathrm{CHA}_{2} \mathrm{DS}_{2}$-VASc score as a predictor of new-onset atrial fibrillation after catheter ablation of typical atrial flutter. Front. Physiol. 11:558. doi: 10.3389/ fphys.2020.00558

Mittal, S., Pokushalov, E., Romanov, A., Ferrara, M., Arshad, A., Musat, D., et al. (2013). Long-term ECG monitoring using an implantable loop recorder for the detection of atrial fibrillation after cavotricuspid isthmus ablation in patients with atrial flutter. Heart Rhythm 10, 1598-1604. doi: 10.1016/j.hrthm.2013.07. 044

Mohanty, S., Natale, A., Mohanty, P., DI Biase, L., Trivedi, C., Santangeli, P., et al. (2015). Pulmonary vein isolation to reduce future risk of atrial fibrillation in patients undergoing typical flutter ablation: results from a randomized pilot study (REDUCE AF). J. Cardiovasc. Electrophysiol. 26, 819-825. doi: 10.1111/ jce. 12688

Nalliah, C. J., Sanders, P., and Kalman, J. M. (2016). Obstructive sleep apnea treatment and atrial fibrillation: a need for definitive evidence. J. Cardiovasc. Electrophysiol. 27, 1001-1010. doi: 10.1111/jce.12981

Nalliah, C. J., Wong, G. R., Lee, G., Voskoboinik, A., Kee, K., Goldin, J., et al. (2021). Sleep apnoea has a dose-dependent effect on atrial remodelling in paroxysmal but not persistent atrial fibrillation: a high-density mapping study. Europace 23, 691-700. doi: 10.1093/europace/euaa275

O’Neal, W. T., Zhang, Z. M., Loehr, L. R., Chen, L. Y., Alonso, A., and Soliman, E. Z. (2016). Electrocardiographic advanced interatrial block and atrial fibrillation risk in the general population. Am. J. Cardiol. 117, 1755-1759.

Park, J. H., Joung, B., Son, N. H., Shim, J. M., Lee, M. H., Hwang, C., et al. (2011). The electroanatomical remodelling of the left atrium is related to CHADS2/CHA2DS2VASc score and events of stroke in patients with atrial fibrillation. Europace 13, 1541-1549. doi: 10.1093/europace/eur135

Poty, H., Saoudi, N., Nair, M., Anselme, F., and Letac, B. (1996). Radiofrequency catheter ablation of atrial flutter. Further insights into the various types of 
isthmus block: application to ablation during sinus rhythm. Circulation 94, 3204-3213. doi: 10.1161/01.cir.94.12.3204

Redline, S., Yenokyan, G., Gottlieb, D. J., Shahar, E., O’Connor, G. T., Resnick, H. E., et al. (2010). Obstructive sleep apnea-hypopnea and incident stroke: the sleep heart health study. Am. J. Respir. Crit. Care Med. 182, 269-277.

Schneider, R., Lauschke, J., Tischer, T., Schneider, C., Voss, W., Moehlenkamp, F., et al. (2015). Pulmonary vein triggers play an important role in the initiation of atrial flutter: initial results from the prospective randomized Atrial Fibrillation Ablation in Atrial Flutter (Triple A) trial. Heart Rhythm 12, 865-871. doi: 10.1016/j.hrthm.2015.01.040

Steinberg, J. S., Romanov, A., Musat, D., Preminger, M., Bayramova, S., Artyomenko, S., et al. (2014). Prophylactic pulmonary vein isolation during isthmus ablation for atrial flutter: the PReVENT AF Study I. Heart Rhythm 11, 1567-1572. doi: 10.1016/j.hrthm.2014.05.011

Tapanainen, J. M., Jurkko, R., Holmqvist, F., Husser, D., Kongstad, O., Makijarvi, M., et al. (2009). Interatrial right-to-left conduction in patients with paroxysmal atrial fibrillation. J. Interv. Card. Electrophysiol. 25, 117-122. doi: 10.1007/ s10840-008-9359-2

Tomson, T. T., Kapa, S., Bala, R., Riley, M. P., Lin, D., Epstein, A. E., et al. (2012). Risk of stroke and atrial fibrillation after radiofrequency catheter ablation of typical atrial flutter. Heart Rhythm 9, 1779-1784.

Tse, G., Wong, C. W., Gong, M., Wong, W. T., Bazoukis, G., Wong, S. H., et al. (2018). Predictive value of inter-atrial block for new onset or recurrent atrial fibrillation: a systematic review and meta-analysis. Int. J. Cardiol. 250, 152-156.

Voight, J., Akkaya, M., Somasundaram, P., Karim, R., Valliani, S., Kwon, Y., et al. (2014). Risk of new-onset atrial fibrillation and stroke after radiofrequency ablation of isolated, typical atrial flutter. Heart Rhythm 11, 1884-1889. doi: 10.1016/j.hrthm.2014.06.038

Conflict of Interest: The authors declare that the research was conducted in the absence of any commercial or financial relationships that could be construed as a potential conflict of interest.

Publisher's Note: All claims expressed in this article are solely those of the authors and do not necessarily represent those of their affiliated organizations, or those of the publisher, the editors and the reviewers. Any product that may be evaluated in this article, or claim that may be made by its manufacturer, is not guaranteed or endorsed by the publisher.

Copyright (c) $2021 \mathrm{Li}$, Xie, Chen, Cao, Tang, Guo, Sun and Ma. This is an open-access article distributed under the terms of the Creative Commons Attribution License (CC BY). The use, distribution or reproduction in other forums is permitted, provided the original author(s) and the copyright owner(s) are credited and that the original publication in this journal is cited, in accordance with accepted academic practice. No use, distribution or reproduction is permitted which does not comply with these terms. 